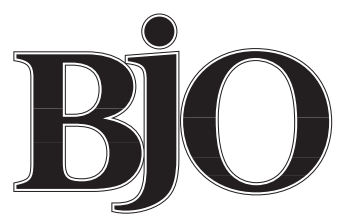

British Journal of Ophthalmology

\title{
Impact of new technologies in ophthalmology
}

New technologies in medicine generally have many origins, some predicted and planned, others serendipitous. The application of the new genetics is an example of the former while the discovery of antibiotics and the intraocular lens might better fit into the latter category. The application of new technology to medicine is set to produce a revolution in health care, in both diagnostics and its management. According to Hendee of the National Institutes of Health, USA " $^{1}$ "biomedical informatics, imaging, and engineering are major forces driving the knowledge revolutions that are shaping the agendas for biomedical research and clinical medicine in the 21 st century." This is a bold statement but there is considerable evidence to support it. Remarkable advances are being made in technologies which will indeed shape the way disease control is managed. Examples include high throughput genetic screening in the post-genomic era using microchip arrays, ${ }^{2}$ biosensor techniques which will allow mass screening for antibodies, ${ }^{3}$ highly sensitive devices to improve management of patients in intensive care, ${ }^{4}$ electronic methods which will guide surgical techniques under computer control, $^{5}$ and improved polymerase chain reaction techniques to assist in the diagnosis of infectious disease. ${ }^{6}$ Developments in proteomics, genomics, and imaging will not only impact on medical care but will provide us with a much greater understanding of normal physiology and, in turn, pathology, and the NIH has called for a greatly increased investment in the development of the three core "tools" identified above. Indeed, a new institute to study systems biology has been established with part of its remit to maximise the advancement and application of the new technologies.

Ophthalmology as a specialty covers the widest of spectra and thus lends itself to the development and application of new technologies. This applies both to the basic sciences of ophthalmology and vision, and to investigative methodology and treatments in clinical ophthalmology. The American Medical Association in its continuation of the global theme issues has proposed new technologies as the theme for 1999 and many journals worldwide are participating in this effort. Thus, the current issue of the $\mathrm{BFO}$ contains a series of articles on a range of new technologies and approaches to ophthalmological problems. All three of Hendee's “tools" are addressed including information technology, imaging, and bioengineering. Thus, informatics forms a significant part of how Black et al ( $\mathrm{p}$ 1215) would see the application of post-genomic knowledge to ophthalmology, while the many aspects of imaging in ophthalmology are addressed by Sharp et al ( $\mathrm{p} \mathrm{1241)}$ and Atta ( $\mathrm{p}$ 1246). Further applications in an experimental model are described by Hossein ( $p$ 1250). Meanwhile, the use of bioengineered phage antibodies has been applied, firstly, to an ophthalmological problem (post-trabeculectomy scarring) because of the unique advantages that the eye offers in evaluating this problem (see paper by Cordeiro et al, p 1219). The novel use of monoclonal antibodies and immunoadhesins in the management of refractory ocular inflammation are also described by Dick and Isaacs ( $p$ 1230), while new intraocular delivery systems for local treatment of ocular disease are presented by Velez and Whitcup ( $p$ 1225). In fact, a whole range of novel biomaterials is likely to expand their use in the eye in several areas, including tissue implants and artificial substrates (see paper by Allan, p 1235). Finally, the advances in refractive surgery represent a very active field of development in ophthalmology, with the holy grail of surgery for presbyopia beckoning (see paper by McDonnell, p 1257). None of these new treatments or investigations would have been possible without the considerable investment in basic science in many fields including physics, engineering, informatics, computer technology, genetics, molecular and cell biology, and many more disciplines. In many cases the potential spinoffs for medicine generally and ophthalmology in particular could not have been predicted and are a strong argument for promoting both directed and "blue skies" research.

Not all problems in ophthalmology are immediately amenable to the new technologies. Some worldwide blinding disease such as trachoma (see paper by Mabey and Bailey, p 1261) will need combinations of new laboratory technologies, for instance for investigative purposes, with imaginative approaches to disease control. These might involve the use of good patient data bases (see paper by Aylward and Palmar, p 1264) with remote monitoring systems such as telemedicine (see paper by Murdoch, p 1254). Bringing together the practical and possible with the new technologies will be the challenge of tomorrow for disease control at the population level, particularly in the developing nations, but also at the level of individual patient care.

JOHN V FORRESTER

1 Hendee WR. Informatics at the National Institutes of Health: a call to action. Am Med Inform Assoc 1999;6:267-71.

2 Cheng J, Fortina P, Surrey S, et al. Microchip-based devices for molecular diagnosis of genetic diseases. Mol Diagn 1996;3:183-200.

3 Swanson SJ, Jacobs SJ, Mytych D, et al. Applications for the new electrochemiluminescent (ECL) and biosensor technologies. Dev Biol Stand 1999;97:135-47.

Ince C, Sinaasappel M. Microcirculatory oxygenation and shunting in sepsis and shock. Crit Care Med 1999;7:1369-77.

5 McDonald MB, Deitz MR, Frantz JM, et al. Photorefractive keratectomy for low-to-moderate myopia and astigmatism with a small-beam, trackerdirected excimer laser. Ophthalmology 1999;106:1481-8.

6 Whitcombe D, Theaker J, Guy SP, et al. Detection of PCR products using self-probing amplicons and fluorescence. Nat Biotechnol 1999;8:804-7.

7 Agrawal A. New institute to study systems biology. Nat Biotechnol 1999;8:743-4. 